\title{
Clostridium difficile and the microbiota
}

\author{
Anna M. Seekatz and Vincent B. Young
}

Division of Infectious Diseases, Department of Internal Medicine, and Department of Microbiology and Immunology, University of Michigan, Ann Arbor, Michigan, USA

\begin{abstract}
Clostridium difficile infection (CDI) is the leading health care-associated illness. Both human and animal models have demonstrated the importance of the gut microbiota's capability of providing colonization resistance against $C$. difficile. Risk factors for disease development include antibiotic use, which disrupts the gut microbiota, leading to the loss of colonization resistance and subsequent CDI. Identification of the specific microbes capable of restoring this function remains elusive. Future studies directed at how microbial communities influence the metabolic environment may help elucidate the role of the microbiota in disease development. These findings will improve current biotherapeutics for patients with CDI, particularly those with recurrent disease.
\end{abstract}

\section{Introduction}

First identified in 1935, Clostridium difficile has become a leading cause of hospital-acquired infections $(1,2)$. Of substantial concern is the increase in severity and morbidity observed within the last decade. Within the US, an estimated 14,000 deaths are attributed to C. difficile infection (CDI) annually, with associated costs between $\$ 1$ billion and $\$ 3$ billion (3). Additionally, initial treatment options fail in $20 \%-30 \%$ of patients, resulting in disease recurrence. These increases in CDI burden have occurred in conjunction with the emergence of hyperendemic strains $(4,5)$.

Since the discovery of $C$. difficile as an etiological agent of antibiotic-associated diarrhea, we have come to appreciate the importance of changes in the indigenous gut microbes, collectively termed the microbiota, in the development of CDI (6). These microbes are estimated to contain 100-fold more genetic potential than our own genome. Thus, the microbiota can provide functions the host alone cannot supply, such as the breakdown of essential nutrients, drug metabolism, immune development, and pathogen resistance (7). Recent technological advances have enhanced our understanding of the microbiota's role in the pathogenesis of CDI. The presence of a healthy gut microbiota is especially relevant in the development of CDI, and future therapeutic strategies will rely on a more complete understanding of the role of the microbiota in disease prevention. In this Review, we will discuss our current knowledge of the role of the microbiota in the pathogenesis of CDI.

\section{CDI pathogenesis: spore exposure and disease development}

An understanding of the pathogenesis of CDI is crucial in disease treatment and prevention (Figure 1). C. difficile is an obligate anaerobe, acquired by the ingestion of spores via the fecal-oral route. These spores can survive even in harsh environmental conditions (8). Because $C$. difficile spores are also resistant to alcohol-based cleaners, spores are especially prevalent in hospital environments and have been detected months after initial exposure $(9,10)$. Infec-

Conflict of interest: The authors have declared that no conflict of interest exists. Reference information: / Clin Invest. 2014;124(10):4182-4189. doi:10.1172/JCI72336. tion with a toxigenic strain of $C$. difficile results in a range of clinical signs and symptoms, from diarrhea and cramping in mild cases to the development of pseudomembranous colitis and even death in severe disease. Although most cases of CDI are health care-related, a percentage of cases occur in the community and appear to be unrelated to antibiotic use or prior health care exposure (Figure 1 and ref. 11). A recent molecular epidemiological study by Eyre et al. that used whole-genome sequencing to track exposure and transmission of C. difficile concluded that one-third of CDI cases were not associated with the hospital (12). Additionally, only one-third of cases were genetically related to each other, suggesting an alternative source of $C$. difficile exposure. $C$. difficile spores have been detected in various environmental sources, including domesticated animals, water sources, and soil (13). Another potential reservoir of $C$. difficile resides in the infant population, in which colonization is estimated to occur in up to $45 \%$ of individuals $(14,15)$. The infant microbiome is distinct from adults', and differences in the microbiome may be important in both colonization and disease resistance $(16,17)$. Although high rates of $C$. difficile colonization are observed in infants, they rarely develop disease. It has been postulated that infants may lack the receptor necessary for disease development (18) or that compounds in human breast milk, such as maternal IgA, may prevent toxin binding (19). Future studies analyzing differences in the colonized infant microbiome may provide useful information about specific microbes that are protective against C. difficile colonization and infection.

Once ingested, spores must germinate and grow into vegetative cells that colonize the gastrointestinal tract. Exposure to spores does not always translate into colonization, as the gastrointestinal environment must be favorable for these events to occur. In vitro studies indicate that germination and outgrowth into the vegetative form depends on the presence of specific primary bile acids, such as taurocholate $(20,21)$. Conversely, other secondary bile acids, such as chenodeoxycholate, inhibit the germination of $C$. difficile spores (22). Microbes within the gastrointestinal tract play a key role in the metabolism of bile acids (23), and it is hypothesized that the modulation of the microbiota community can impact metabolite availability. Giel et al. determined that cecal extracts from antibiotic-treated mice contained elevated 


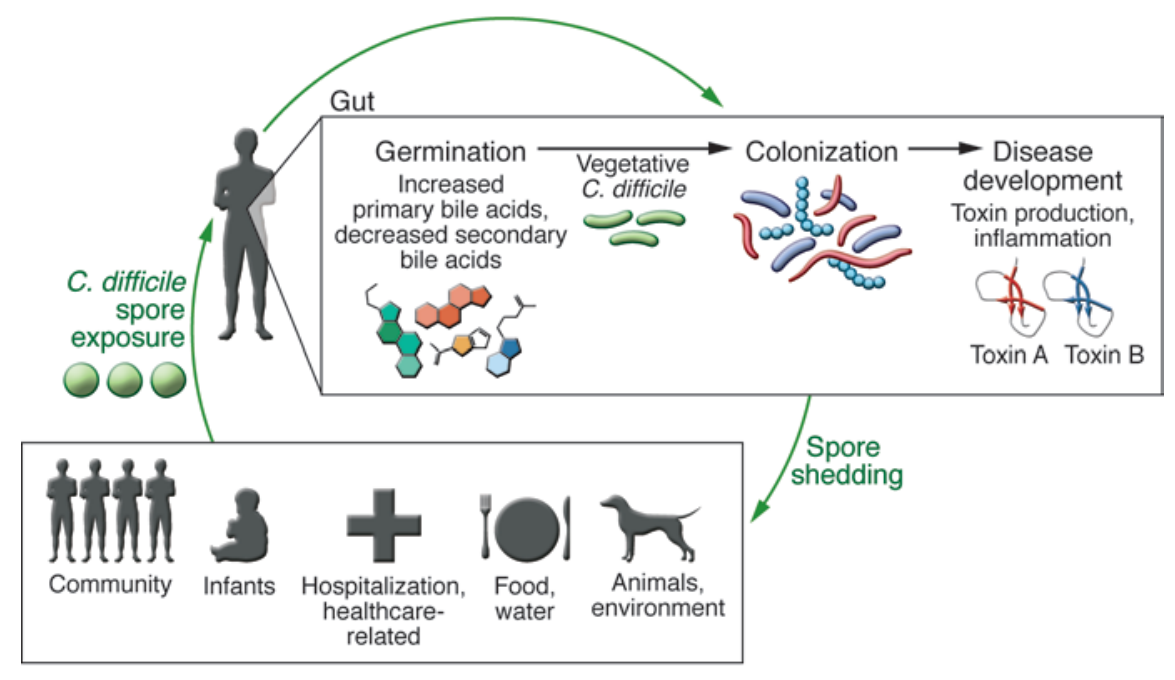

Figure 1. CDI pathogenesis. Development of disease is dependent on different stages of the C. difficile life cycle. Initial spore exposure from various sources does not necessarily result in disease, particularly in a healthy individual. A healthy, diverse microbiota is capable of interfering with C. difficile spore germination and vegetative growth. However, if the metabolic and microbial environment of the gut has been perturbed, spore germination, vegetative outgrowth, and toxin production will occur. Epithelial damage, inflammation, and clinically overt disease will result from toxin production. Sporulation of $C$. difficile, release of spores into the environment, and transmission to new hosts perpetuates the infectious cycle. levels of bile salts and promoted spore germination, while cecal extracts from untreated mice did not (24). Similarly, Theriot et al. observed significant shifts in the metabolome of antibiotic-treated mice that correlated to changes in the microbial community structure (25). Both human and in vivo mouse studies have revealed the importance of bile acids in $C$. difficile germination and continue to enhance our knowledge about spore germination (25-27).

Once colonized, C. difficile can lead to toxin-mediated inflammation and disease. $C$. difficile produces 2 major toxins responsible for disease, the large clostridial toxins A and B (TcdA and TcdB). These toxins, produced during the stationary phase of vegetative growth, are largely responsible for the damage to the mucosal epithelium and induction of an inflammatory response (28). Another toxin, the $C$. difficile binary toxin (CDT), has been observed to disrupt the actin cytoskeleton, and some studies suggest its presence may increase strain virulence $(29,30)$; however, its presence does not always correlate with disease severity (31). The dynamic life cycle of $C$. difficile is complex, and multiple host factors may be involved at each step (Figure 2). Since spore exposure and $C$. difficile colonization does not necessarily result in clinical disease, the gastrointestinal microbial community and the host may provide an important role in disease development throughout the life cycle of $C$. difficile.

\section{Disruption of the microbiome and CDI risk factors}

At the core of CDI pathogenesis is disruption of the microbiota (Figure 2). A healthy gut microbiota is necessary for protection against pathogen colonization, termed colonization resistance (32). An undisrupted microbiota is capable of resisting colonization by pathogens, and multiple mechanisms have been suggested for why disruption of the microbiota leads to loss of colonization resistance, including competition for nutrients, ecological competition, and niche exclusion (33). While many risk factors associated with CDI can result in disruption of the microbiota, the most commonly associated factor is antibiotic use. Both short-term and long-term changes have been observed in the gut microbiota following antibiotic use. Decreases in the gut microbiota diversity are detectable within days of antibiotic use, although compositional changes may be dependent on the antibiotic used as well as the underlying microbiota community of the individual $(34,35)$. Reconstitution of the microbial diversity does occur following cessation of antibiotic use, but can also result in an altered community structure. Dethlefsen et al. found that although recovery began within weeks of ciprofloxacin cessation, the new community did not necessarily include all community members observed before antibiotic use (36). The classes of antibiotics associated with development of CDI include clindamycin, cephalosporins, and penicillins (37).

Increasing age, another known risk factor for development of CDI, has also been observed to impact the structure of the microbiome. The human gut microbiome undergoes extreme changes throughout life, and it is not surprising that shifts in the microbial composition have been observed in elderly people $(38,39)$. While the gut microbiome of healthy adults appears relatively stable over time, the gut microbiome of the elderly has been observed to be in flux and less diverse. Microbiota studies in elderly cohorts have observed a decrease in protective species, such as Bifidobacteria and some Firmicutes members, as well as an increase in Bacteroidetes and more detrimental species, such as Proteobacteria (38, 40). These changes appear to partially accompany degradation of the immune system in older age, termed immunosenescence. Considering these observations, it is not surprising that the rate of CDI is higher for people ages 65 and over and accounts for the majority of diarrheal cases in long-term living facilities (41-43). While it is possible that age is an independent risk factor for CDI, advancing age is also associated with increased antibiotic use, more frequent hospital visits, and the development of illnesses in general, all of which impact $C$. difficile susceptibility.

Other risk factors associated with the development of CDI also have the potential to disrupt the microbiota (Figure 2). The use of proton pump inhibitors (PPIs), particularly in conjunction with antibiotics, has been correlated with higher CDI incidence in some studies $(44,45)$. It is hypothesized that PPIs, which generally increase the $\mathrm{pH}$ of the stomach, may affect other gastrointestinal sites and are thus capable of modulating the microbiota (46). Indeed, in vitro studies have shown that PPIs can affect the growth of Lactobacillus, a common resident of the mouth and gut (47). 
A

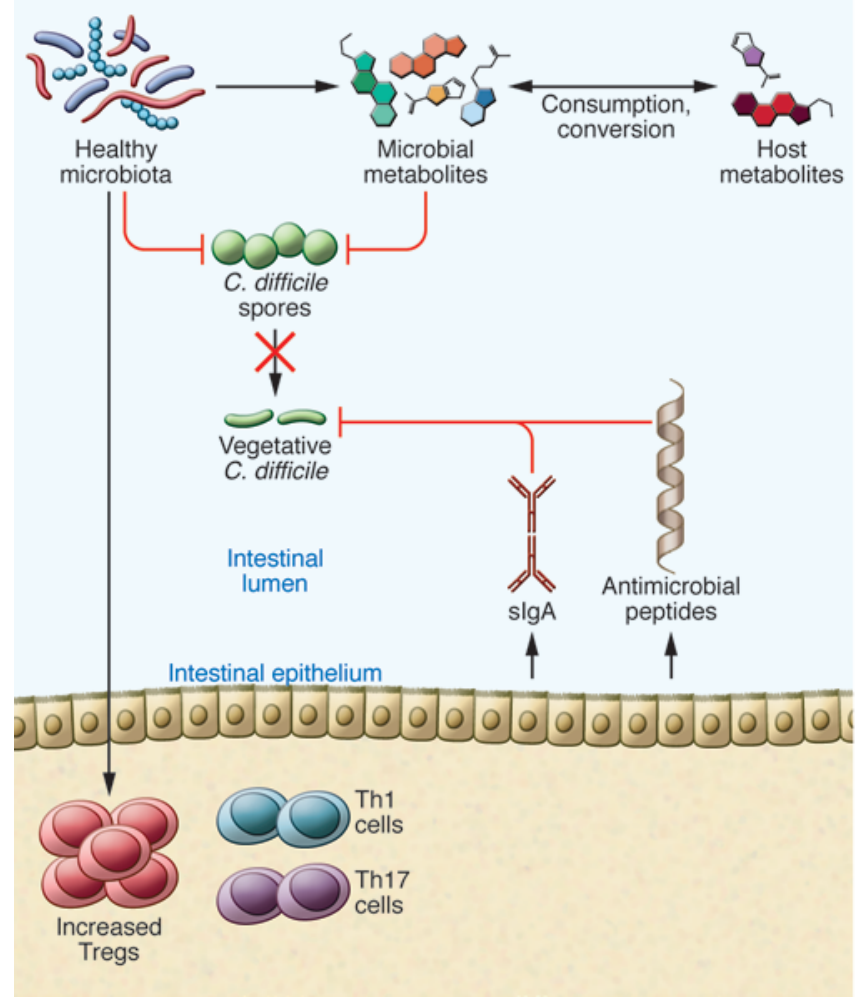

B

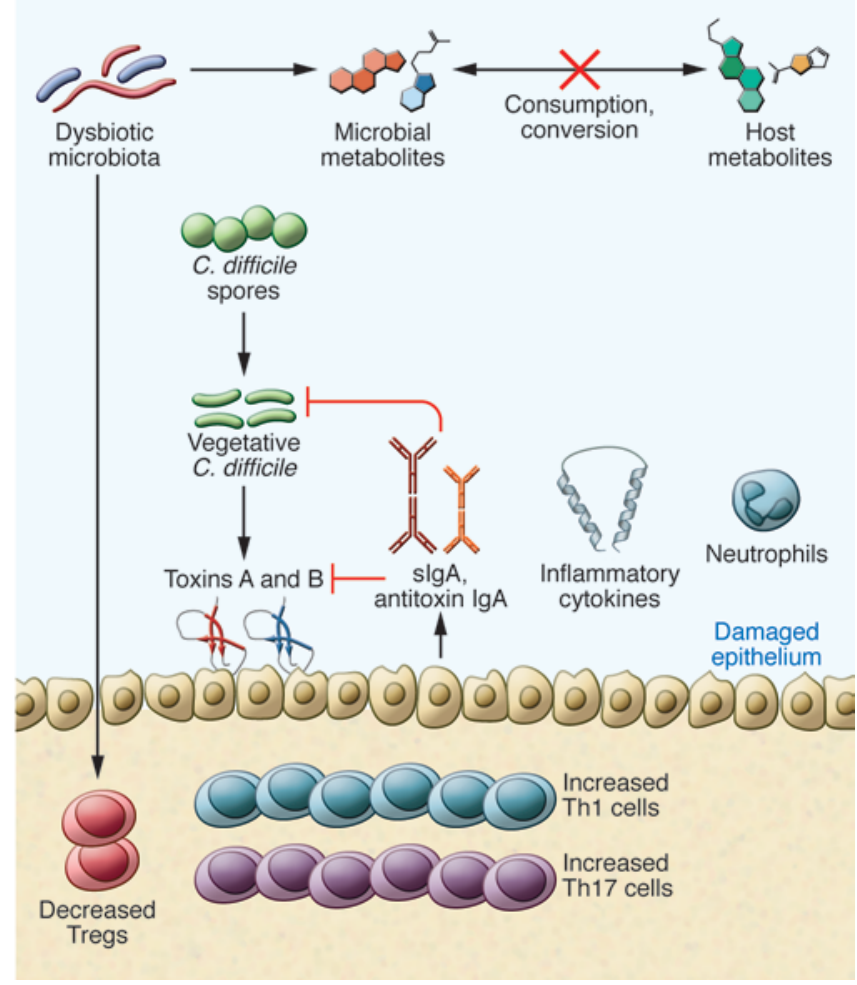

Figure 2. Proposed mechanisms of the microbiota on pathogen resistance and the host during CDI. (A) Both microbial and host factors can inhibit germination and growth of $C$. difficile. A healthy microbiota is capable of consuming both microbial and host-generated metabolites, limiting the growth of $C$. difficile. Cross-talk between the microbiota and the host immune system results in a regulated immune response. Furthermore, the microbiota can stimulate production of antimicrobial peptides and secretory $\operatorname{lgA}(\mathrm{s} \lg A$ ) that can maintain the composition of the microbiota. (B) Disruption of the microbiota, due to factors such as antibiotic use, drugs, diet, or inflammation, can lead to the development of CDI. A dysbiotic microbiota can result in the loss of colonization resistance due to changes in the structural and/or metabolic environment. The loss of specific community members potentially affects the levels of microbial and host-generated metabolites, resulting in a different functional state that promotes spore germination and vegetative outgrowth. A dysbiotic microbiota may also result in an imbalanced immune response through the loss of immune regulation and a proinflammatory state, both of which may affect disease development. Toxin production by vegetative $C$. difficile can stimulate the production of inflammatory cytokines, neutrophils, and antitoxin antibodies.

Patients suffering from other gastrointestinal disease may also be more likely to acquire $C$. difficile. Inflammatory bowel disease (IBD) has been linked to more severe disease outcome and is increasingly found to be a risk factor for CDI $(48,49)$. A decreased diversity of Firmicutes and Bacteroidetes has been observed in the microbiota of IBD patients (50). Additionally, the microbiota of patients with IBD has been associated with the presence of multiple potentially pathogenic bacteria, mainly within the Proteobacteria phylum $(51,52)$. However, how these communities impact susceptibility to $C$. difficile itself appears complex.

The host immune response also has the capability to modulate the microbiota. The observation that IBD can aggravate disease outcome of CDI suggests that inflammation may contribute to the development of CDI. Inflammatory products, such as the antimicrobial peptides lipocalin-2 and calprotectin, limit the availability of nutrients in the gut environment, potentially impacting the growth of surrounding microbes $(53,54)$. These changes may provide a more amenable environment for $C$. difficile colonization and subsequent CDI. In addition to inflammation-driven microbiome changes, other host-driven immune responses may be involved in disease outcome. The type of gut community can potentially affect the resident mucosal IgA repertoire (55), and a reduction in IgA-producing cells has been observed in colonic biopsies of patients with recurrent disease (56). Various microbes have also been observed to impact subsets of $\mathrm{T}$ cells, such as the induction of Tregs by Clostridium species (57) and Th17 cell differentiation by segmented filamentous bacteria (58). Modulation of these microbial populations, such as after antibiotics, is likely to influence pathogen colonization. Russell et al. observed that vancomycin treatment in mice resulted in a decreased abundance of Bacteroides species in the gut, which was correlated with a decreased abundance of colonic Tregs (59). It is likely that host factors, modulated by antibiotics or not, affect disease outcome.

The Human Microbiome Project revealed the extent of variation within the "healthy" or "normal" gut microbiota (60). These observations have markedly fine-tuned our definition of dysbiosis within the gut microbiota and continue to complicate the causal role of the microbiome in disease development. Moving forward, we hope to answer more mechanistic questions about the risk factors correlated with disruption of the microbiota and the development of CDI. 
Table 1. Summary of observed protective microbial taxa (negatively correlated to $C$. difficile colonization) and susceptible microbial taxa (positively correlated to $C$. difficile colonization) in human studies investigating changes in the microbiota community and CDI

Study cohort

\begin{tabular}{|c|c|}
\hline & $\begin{array}{l}\text { Protective: negatively correlated } \\
\text { to } C \text {. difficile colonization }\end{array}$ \\
\hline Infants & Bifidobacterium longum (17) \\
\hline \multirow[t]{5}{*}{ Elderly } & Bacteroides spp. $(61,62)$ \\
\hline & Prevotella spp. $(61,63)$ \\
\hline & Bifidobacterium spp. (61) \\
\hline & Enterococcaceae (63) \\
\hline & Leuconostocaceae (63) \\
\hline
\end{tabular}

Adults (variable age)
Fecal microbiota transplantation
patients

Ruminococcaceae $(64,65)$ Lachnospiraceae $(64,65)$ Bacteroides spp. (65) Porphyromonadaceae (65) Bifidobacterium spp. (98) Methanobrevibacter spp. (98) Bacteroides spp. (27, 74-77, 80, 94) Parabacteroides spp. (77) Alistipes (77)

Ruminococcaceae $(75,77,78)$

Clostridium cluster IV $(76,80)$

Clostridium cluster XIVa $(64,76)$ : Faecalibacterium prausnitzii Roseburia intestinalis Butyrivibrio crossotus Eubacterium rectale (80) Lachnospiraceae $(77,78)$ Peptostreptococcaceae (78) Verrucomicrobiaceae (77)

\section{Observed correlation to CDI}

Susceptible: positively correlated
to C. difficile colonization
Ruminococcus gnavus $(17)$
Klebsiella pneumonia $(17)$
Lactobacilli (61)
Aerococcaceae (61)
Enterobacteria (61)
Enterococcal gr. (61)
Clostridiales:
$\quad$ Clostridium spp. $(61,63)$
Enterobacteriaceae $(64,65)$
Enterococcus $(64,65)$
Lactobacillus $(64)$
Erysipelotrichaceae $(65)$

Lactobacillus spp. $(75,77,80)$
Streptococcaceae $(75,78)$
Enterobacteriaceae:
Enterobacter aerogenes $(76,77,80)$
Klebsiella spp. $(76,78,80)$
Proteus spp. $(80)$
Veillonella $(27,75,77,80)$
Enterococcus spp. $(78,80)$
Salmonella spp. $(77)$
Sutterella spp. $(80)$
Verrucomicrobia $(27)$

tes) (61). Healthy adults were also more likely to have more Bifidobacteria and Bacteroides compared with either elderly population. More recent studies using high-throughput sequencing of the $16 \mathrm{~S}$ rRNA gene have provided a more indepth look at the community structure in C. difficile-positive people (62-64). In a cohort of elderly patients, Rea et al. observed that patients with active CDI harbored a less diverse gut microbiota compared with their healthy counterparts (63). Increases in Lactobacillaceae and Enterobacteriaceae, but decreases in Enterococcaceae, were observed in patients positive for $C$. difficile. Similar results have been reported in studies comparing healthy adults with both CDI and non-CDI patients with diarrhea $(64,65)$. Compared with healthy adults, both groups had significantly less diverse communities, particularly a less diverse Firmicutes population. Lachnospiraceae, Ruminococcaceae, and Bacteroidaceae families dominated the communities observed in healthy people. Interestingly, non-CDI and CDI patients with active diarrhea had strikingly similar communities, suggesting that diarrhea or inflammation itself may be correlated to a particular microbiota community. Furthermore, most CDI samples have been collected throughout antibiotic use, which may simplify the community structure observed. While results from different studies are concordant, the interindividual differ-

The microbiota mediates colonization resistance against $C$. difficile

While the general importance of the gut microbiota in CDI development is well established, the exact microbes responsible for protection or susceptibility remain elusive (Table 1). The lack of prospective human samples before CDI has complicated the identification of microbiome signatures that correlate with protection. Several cross-sectional studies have compared samples from CDI patients with samples from both healthy and non-CDI patients with diarrhea. Infants represent an interesting population to study, as most infants can be colonized but do not develop disease. Rousseau et al. observed that $C$. difficile colonization in infants was accompanied by the presence of Ruminococcus and Klebsiella species, while Bifidobacterium appeared protective against colonization (17). Several studies have also compared samples from elderly populations, which are more susceptible. Using nonsequencing methods, Hopkins et al. observed that elderly patients with CDI had higher counts of Enterobacteriaceae (Proteobacteria), Enterococcus, and Lactobacillus (both Firmicutes), whereas healthy elderly patients harbored more diverse Bacteroides strains (Bacteroide- ences in the gut microbiota across the studies are apparent. Both host factors and environmental influences on the gut microbiota and disease itself complicate the identification of specific microbial markers responsible for disease protection.

Similar general observations have been made in murine models, in which environmental and genetic variances can be more controlled. As in humans, antibiotic administration decreases the diversity of the gut microbiota in mice, rendering them more susceptible to multiple enteric diseases, including CDI. Lawley et al. observed that a reduced microbial diversity in the mouse gut, dominated by Enterococci and Proteobacteria, following clindamycin treatment induced disease and shedding of contagious spores (66). Another clindamycin-based model of CDI in mice reported similar decreases in Enterobacteriaceae members, as well as differences in the recovery of the communities with and without CDI (67). Reeves et al. found that susceptible mice were dominated by Lactobacillaceae and Enterobacteriaceae families before infection following treatment with cefoperazone, clindamycin, or a multi-antibiotic cocktail (68). Conversely, Lachnospiraceae members dominated animals that remained resistant to CDI. A 
follow-up study based on these observations observed that mice colonized by Lachnospiraceae isolates, but not those colonized by E. coli isolates, exhibited decreased C. difficile colonization and less severe disease (69). Despite differences at lower taxonomic levels in the gut microbiota between humans and mice, murine models have provided a more testable way of identifying components protective in CDI development.

One of the difficulties that have hindered the identification of the specific community members that confer colonization resistance is the inherent interindividual variability of the microbiota observed in the human population. Furthermore, the identification of the same type of microbe does not guarantee that the microbe will have an identical genetic function, nor does the identification of different microbes exclude the possibility of similar functions within a community. Instead, recent studies have proposed that the metabolic, or functional, environment may be more indicative of susceptible states compared with community structure. A recent study by Theriot et al. suggests that antibiotic-induced changes that render mice susceptible to $C$. difficile are better reflected in terms of metabolic changes rather than changes in the microbial community composition (25). Although antibiotic-treated mice eventually recovered colonization resistance, their community composition was altered compared with their preantibiotic community, suggesting that functional changes rather than community changes are important in maintaining resistance to CDI. Further studies investigating how different communities may provide similar functions will be necessary to elucidate the structure-function relationship of the microbiome during infection.

\section{Recurrent CDI: incomplete recovery of the microbiota}

A major concern in CDI treatment is recurrence of disease following a seemingly successful response to standard therapy consisting of antibiotics known to suppress growth of $C$. difficile, metronidazole and/or vancomycin. Recurrence is estimated to occur in $20 \%-30 \%$ of CDI patients; after each incidence, the chance of recurrence increases (70). Although it is unknown why recurrence occurs in some patients and not others, risk factors for recurrent CDI include the use of non-CDI antibiotics following an initial episode, as well as increased age and disease severity (71). It is hypothesized that antibiotic treatment interferes with the ability of the gut microbiota to recover fully and reestablish colonization resistance in some individuals. Alternatively, recurrence could reflect failure of the host to mount a protective immune response against $C$. difficile.

Few studies have investigated structural signatures in the microbiome that potentially lead to recurrence. Most human CDI studies have not included a longitudinal aspect to their studies or have not distinguished patients with multiply recurrent CDI. Chang et al. observed that the microbiota community was less diverse in recurrent patients than in those with a single case of CDI, suggesting that recurrence could be predicted on the basis of the microbiota community present during infection (72). While some studies comparing non-CDI and CDI samples have included secondary recurrent samples in their analysis, none were able to identify particular aspects unique to patients with recurrent CDI (63).

Interestingly, much of our knowledge about the microbiota during recurrent CDI comes from an alternative treatment meth- od, fecal microbiota transplantation (FMT). Use of FMT, or fecal bacteriotherapy, has become a popular, highly effective treatment method for recurrent CDI. The success rate of FMT is up to $92 \%$ in multiply recurrent CDI patients, depending on the protocol used (73). It is presumed that FMT is capable of restoring the microbiota and colonization resistance. However, as with the identification of which specific microbes may indicate susceptibility to CDI, the microbes responsible for restoring colonization resistance have not been specifically identified. Earlier studies using either culturebased or PCR-based methods have reported the recovery of Bacteroides species and detection of more Firmicutes in culture, only after the FMT procedure, along with successful clinical recovery $(74,75)$. Recent studies using $16 \mathrm{~S}$ rRNA surveys have observed that after FMT, diversity of the gut microbiota increases and resembles the donor's (76-78). Recovery of both Firmicutes and Bacteroides has been observed. Additionally, the level of Proteobacteria, generally found at high levels within patients with active CDI, decreases after FMT. Interestingly, the dominant donor microbes classified at either at the genus level or the operational taxonomic unit level are observed to be prevalent within the recipient for only days following FMT $(77,79)$. The microbes that are found to be dominant within recipients in the long term appear to be recipient-specific, even if the community is more similar to the donor's than before FMT. These observations suggest that direct colonization by the donor's microbes is not necessarily what accounts for recovery of the microbiota community following FMT.

As with the identification of communities that render an individual more susceptible to $C$. difficile during initial infection, the functional state of the environment may be more telling than the structure. Indeed, human microbiome studies of CDI have observed a decrease in butyrate-producing microbial taxa and have postulated that the abundance of microbial by-products, such as short-chain fatty acids, may be indicative of susceptibility to $\operatorname{CDI}(64,80,81)$. A recent study by Weingarden et al. observed high concentrations of primary bile acids in patients with recurrent CDI (27). Following FMT, the concentration of secondary bile acids, undetected in pre-FMT samples, was increased and was found at a relative abundance close to that of healthy donors. These results are in agreement with the in vitro and in vivo mouse studies that have previously observed that secondary bile acids, such as lithocholic or deoxycholic acid, inhibit $C$. difficile growth $(20,25)$. Although the bacterial community is responsible for producing the metabolic environment, it is possible that several types of bacterial communities with similar functions may be capable of the same metabolic outcome, and that structure alone may not be enough to determine recurrence risk (82).

\section{C. difficile: future therapeutics and research directions}

In addition to standard therapy or FMT, other treatment methods for CDI have been explored. Ideally, therapy would be effective against $C$. difficile but fail to globally affect the indigenous gut microbiota. Antibiotics other than vancomycin or metronidazole, such as fidaxomicin, tigecycline, and rifampicin, have been used to treat severe or recurrent CDI $(83,84)$. Fidaxomicin was also shown to exert little effect on Bacteroides counts, which may be advantageous in preserving colonization resistance (85). Tigecycline has 
been observed to inhibit toxin production and growth in mice and has been used to treat severe disease in humans $(86,87)$. However, even antibiotics that have intrinsic capability against $C$. difficile are able to change the microbiota, potentially resulting in a loss of colonization resistance (88). The future of CDI treatment will likely include nonantibiotic therapeutic approaches against CDI, which are advantageous since they may be less likely to perturb the microbiota in a detrimental manner. One option is to treat the primary cause of disease development in CDI, toxin activity by toxins A and B. Serum IgG antibodies against toxins A and B have been correlated with protection in human studies $(89,90)$. Both passive and active immunization strategies against toxins have been explored as potential methods to treat $C$. difficile (91). Drugs that bind toxin in vitro, such as tolevamer, have also been used in human trials, but with limited success (92). Although antitoxin therapies may prevent the effects of toxin and disease development, they do not prevent $C$. difficile colonization or potential spore transmission.

Like FMT, therapies involving live microorganisms have great promise in CDI treatment and prevention. Synthetic mixes of bacteria have been suggested as potential biotherapeutic approaches to treating CDI as an alternative to fecal transplantation directly from a donor. Although donors are generally screened for known pathogens before FMT, there is still a risk of transmission of unknown pathogens or unknown risks associated with the microbiota. A synthetic mix provides control over many safety issues compared with direct fecal matter, such as reducing the potential risk of pathogen transmission and providing more reproducible control over the types of bacteria contained in the mixture. Lawley et al. were able to identify a population of 6 different bacteria that were efficient at clearing CDI in mice (93). In humans, Petrof et al. reported successful treatment of recurrent CDI in 2 patients with a community consisting of 33 isolates from a healthy donor (94). Furthermore, if the functional aspects rather than the community itself can lead to colonization resistance, formulation of an effective biotherapeutic option may include organisms that are capable of providing a metabolic environment that promotes the growth of existing healthy microbes, such as Bacteroides or Firmicutes, rather than fully replacing the community favorable to $C$. difficile. These data have generated great interest in creating commercial biotherapeutics to replace FMT, potentially leading to the devel- opment of prebiotics or prescribed diets instead of bacterial communities to enhance an environment resistant to $C$. difficile outgrowth and/or colonization.

The observation that asymptomatically colonized patients have a reduced risk of developing CDI has prompted research into using nontoxigenic strains as preventative therapy against $C$. difficile (95). Recently, Nagaro et al. observed that hamsters infected with nontoxigenic strains were protected from infection with the hyperendemic BI/NAP1/027 strain, which is usually $100 \%$ fatal in hamsters (96). Nontoxigenic strains have also been used safely in studies of volunteer patients in prevention of recurrent $C$. difficile (97). Both the generation of a protective immune response by the host and competitive niche theories have been hypothesized to explain these results. However, the potential for gene transfer of virulence factors and antibiotic resistance among nontoxigenic and toxigenic strains is a concern.

\section{Conclusion}

Although a basic picture of CDI pathogenesis is known, a better understanding of the microbiota's role in disease prevention is necessary. The role of the gut microbiota is integral throughout the life cycle of $C$. difficile from spore transmission, germination, and growth, into disease development. Although our understanding about the complexity of disease development and transmission has improved in recent decades, we still lack knowledge on which components are crucial points of interruption. The development of future therapeutics to treat disease and minimize transmission depends on expanding our current knowledge.

\section{Acknowledgments}

The NIH grant U19AI090871 (to V.B. Young) and the Michigan Gastrointestinal Peptide Research Center (P3ODK034933) provided funding for this research. We thank Mark Mazaitis for his consultation on the figure concepts.

Address correspondence to: Vincent B. Young, Department of Internal Medicine, Division of Infectious Diseases, University of Michigan Medical School, 1520B MSRB I, 1500 W. Medical Center Drive, Ann Arbor, Michigan 48109, USA. Phone: 734.764.2237; E-mail: youngvi@umich.edu.
1. Hall IC, O'Toole E. Intestinal flora in new-born infants with a description of a new pathogenic anaerobe, Bacillus difficilis. Am J Dis Child. 1935;49(2):390-402.

2. Ananthakrishnan AN. Clostridium difficile infection: epidemiology, risk factors and management. Nat Rev Gastroenterol Hepatol. 2011;8(1):17-26.

3. Dubberke E. Clostridium difficile infection: the scope of the problem. J Hosp Med. 2012;

7(suppl 3):S1-S4.

4. Loo VG, et al. A predominantly clonal multi-institutional outbreak of Clostridium difficile-associated diarrhea with high morbidity and mortality. N Engl JMed. 2005;353(23):2442-2449.

5. Pépin J, et al. Clostridium difficile-associated diarrhea in a region of Quebec from 1991 to 2003: a changing pattern of disease severity. CMAJ. 2004;171(5):466-472.
6. Bartlett JG, Moon N, Chang TW, Taylor N, Onderdonk AB. Role of Clostridium difficile in antibiotic-associated pseudomembranous colitis. Gastroenterology.1978;75(5):778-782.

7. Britton RA, Young VB. Role of the intestinal microbiota in resistance to colonization by Clostridium difficile. Gastroenterology. 2014;146(6):1547-1553.

8. Underwood S, et al. Characterization of the sporulation initiation pathway of Clostridium difficile and its role in toxin production. J Bacteriol. 2009;191(23):7296-7305.

9. $\mathrm{Kim} \mathrm{KH}$, et al. Isolation of Clostridium difficile from the environment and contacts of patients with antibiotic-associated colitis. JInfect Dis. 1981;143(1):42-50.

10. Riggs MM, Sethi AK, Zabarsky TF, Eckstein EC, Jump RL, Donskey CJ. Asymptomatic carriers are a potential source for transmission of epidemic and nonepidemic Clostridium difficile strains among long-term care facility residents. Clin Infect Dis. 2007;45(8):992-998.

11. Chitnis AS, et al. Epidemiology of community-associated Clostridium difficile infection, 2009 through 2011. JAMA Intern Med. 2013;173(14):1359-1367.

12. Eyre DW, Wilcox MH, Walker AS. Diverse sources of C. difficile infection. N Engl JMed. 2014;370(2):183-184.

13. al Saif N, Brazier JS. The distribution of Clostridium difficile in the environment of South Wales. JMed Microbiol. 1996;45(2):133-137.

14. Rousseau C, Poilane I, De Pontual L, Maherault AC, Le Monnier A, Collignon A. Clostridium difficile carriage in healthy infants in the community: a potential reservoir for pathogenic strains. 
Clin Infect Dis. 2012;55(9):1209-1215.

15. Collignon A, Ticchi L, Depitre C, Gaudelus J, Delmée M, Corthier G. Heterogeneity of Clostridium difficile isolates from infants. Eur J Pediatr. 1993;152(4):319-322.

16. Schutze GE, et al. Clostridium difficile infection in infants children. Pediatrics. 2013;131(1):196-200.

17. Rousseau C, Levenez F, Fouqueray C, Doré J, Collignon A, Lepage P. Clostridium difficile colonization in early infancy is accompanied by changes in intestinal microbiota composition. JClin Microbiol. 2011;49(3):858-865.

18. Eglow R, et al. Diminished Clostridium difficile toxin A sensitivity in newborn rabbit ileum is associated with decreased toxin A receptor. JClin Invest. 1992;90(3):822-829.

19. Rolfe RD, Song W. Immunoglobulin and nonimmunoglobulin components of human milk inhibit Clostridium difficile toxin A-receptor binding. J Med Microbiol. 1995;42(1):10-19.

20. Sorg JA, Sonenshein AL. Inhibiting the initiation of Clostridium difficile spore germination using analogs of chenodeoxycholic acid, a bile acid. J Bacteriol. 2010;192(19):4983-4990.

21. Heeg D, Burns DA, Cartman ST, Minton NP. Spores of Clostridium difficile clinical isolates display a diverse germination response to bile salts. PLoS One. 2012;7(2):e32381.

22. Sorg JA, Sonenshein AL. Chenodeoxycholate is an inhibitor of Clostridium difficile spore germination. J Bacteriol. 2009;191(3):1115-1117.

23. Macfarlane GT, Macfarlane S. Bacteria, colonic fermentation, and gastrointestinal health. JAOAC Int. 2012;95(1):50-60.

24. Giel JL, Sorg JA, Sonenshein AL, Zhu J. Metabolism of bile salts in mice influences spore germination in Clostridium difficile. PLoS One. 2010;5(1):e8740.

25. Theriot CM, et al. Antibiotic-induced shifts in the mouse gut microbiome and metabolome increase susceptibility to Clostridium difficile infection. Nat Commun. 2014;5:3114.

26. Howerton A, Patra M, Abel-Santos E. A new strategy for the prevention of Clostridium difficile infection. J Infect Dis. 2013;207(10):1498-1504.

27. Weingarden AR, et al. Microbiota transplantation restores normal fecal bile acid composition in recurrent Clostridium difficile infection. Am J Physiol Gastrointest Liver Physiol. 2013;306(4):G310-G319.

28. Voth DE, Ballard JD. Clostridium difficile toxins: mechanism of action and role in disease. Clin Microbiol Rev. 2005;18(2):247-263.

29. Gerding DN, Johnson S, Rupnik M, Aktories K. Clostridium difficile binary toxin CDT: Mechanism, epidemiology, and potential clinical importance. Gut Microbes. 2014;5(1):15-27.

30. Stewart DB, Berg AS, Hegarty JP. Single nucleotide polymorphisms of the tcdC gene and presence of the binary toxin gene predict recurrent episodes of Clostridium difficile infection [published online ahead of print December 26, 2013]. Ann Surg. doi:10.1097/ SLA.0000000000000469.

31. Goldenberg SD, French GL. Lack of association of tcdC type and binary toxin status with disease severity and outcome in toxigenic Clostridium difficile. J Infect. 2011;62(5):355-362.
32. Vollaard EJ, Clasener HA. Colonization resistance. Antimicrob Agents Chemother. 1994;38(3):409-414.

33. Britton RA, Young VB. Interaction between the intestinal microbiota and host in Clostridium difficile colonization resistance. Trends Microbiol. 2012;20(7):313-319.

34. Dethlefsen L, Huse S, Sogin ML, Relman DA. The pervasive effects of an antibiotic on the human gut microbiota, as revealed by deep $16 \mathrm{~S}$ rRNA sequencing. PLOS Biol. 2008;6(11):e280.

35. Antonopoulos DA, Huse SM, Morrison HG, Schmidt TM, Sogin ML, Young VB. Reproducible community dynamics of the gastrointestinal microbiota following antibiotic perturbation. Infect Immun. 2009;77(6):2367-2375.

36. Dethlefsen L, Relman DA. Incomplete recovery and individualized responses of the human distal gut microbiota to repeated antibiotic perturbation. Proc Natl Acad Sci USA. 2011;108(suppl 1):4554-4561.

37. McFarland LV. Antibiotic-associated diarrhea: epidemiology, trends and treatment. Future Microbiol. 2008;3(5):563-578.

38. Claesson MJ, et al. Composition, variability, and temporal stability of the intestinal microbiota of the elderly. Proc Natl Acad Sci USA. 2011;108(suppl 1):4586-4591.

39. Biagi E, et al. Through ageing, and beyond: gut microbiota and inflammatory status in seniors and centenarians. PLoS One. 2010;5(5):e10667.

40. Hopkins MJ, Sharp R, Macfarlane GT. Age and disease related changes in intestinal bacterial populations assessed by cell culture, $16 \mathrm{~S}$ rRNA abundance, community cellular fatty acid profiles. Gut. 2001;48(2):198-205.

41. Simor AE, Bradley SF, Strausbaugh LJ, Crossley $\mathrm{K}$, Nicolle LE, SHEA Long-Term-Care Committee. Clostridium difficile in long-term-care facilities for the elderly. Infect Control Hosp Epidemiol. 2002;23(11):696-703.

42. Keller JM, Surawicz CM. Clostridium difficile infection in the elderly. Clin Geriatr Med. 2014;30(1):79-93.

43. Aronsson B, Möllby R, Nord CE. Antimicrobial agents and Clostridium difficile in acute enteric disease: epidemiological data from Sweden, 1980-1982. J Infect Dis. 1985;151(3):476-481.

44. Dial S, Delaney JA, Barkun AN, Suissa S. Use of gastric acid-suppressive agents and the risk of community-acquired Clostridium difficile-associated disease. JAMA. 2005;294(23):2989-2995

45. Dial S, Delaney JA, Schneider V, Suissa S. Proton pump inhibitor use and risk of communityacquired Clostridium difficile-associated disease defined by prescription for oral vancomycin therapy. CMAJ. 2006;175(7):745-748.

46. Vesper BJ, Jawdi A, Altman KW, Haines GK 3rd, Tao L, Radosevich JA. The effect of proton pump inhibitors on the human microbiota. Curr Drug Metab. 2009;10(1):84-89.

47. Altman KW, et al. Effect of proton pump inhibitor pantoprazole on growth and morphology of oral Lactobacillus strains. Laryngoscope. 2008;118(4):599-604.

48. Nguyen GC, Kaplan GG, Harris ML, Brant SR. A national survey of the prevalence and impact of Clostridium difficile infection among hospitalized inflammatory bowel disease patients. Am J
Gastroenterol. 2008;103(6):1443-1450.

49. Berg AM, Kelly CP, Farraye FA. Clostridium difficile infection in the inflammatory bowel disease patient. Inflamm Bowel Dis. 2013;19(1):194-204.

50. Manichanh C, et al. Reduced diversity of faecal microbiota in Crohn's disease revealed by a metagenomic approach. Gut. 2006;55(2):205-211.

51. Nagalingam NA, Lynch SV. Role of the microbiota in inflammatory bowel diseases. Inflamm Bowel Dis. 2012;18(5):968-984.

52. Ohkusa T, Sato N, Ogihara T, Morita K, Ogawa M, Okayasu I. Fusobacterium varium localized in the colonic mucosa of patients with ulcerative colitis stimulates species-specific antibody. J Gastroenterol Hepatol. 2002;17(8):849-853.

53. Faber F, Bäumler AJ. The impact of intestinal inflammation on the nutritional environment of the gut microbiota. Immunol Lett. 2014; pii:S0165-2478(14)00083-2.

54. Deriu E, et al. Probiotic bacteria reduce salmonella typhimurium intestinal colonization by competing for iron. Cell Host Microbe. 2013;14(1):26-37.

55. Macpherson AJ, McCoy KD. Stratification and compartmentalisation of immunoglobulin responses to commensal intestinal microbes. Semin Immunol. 2013;25(5):358-363.

56. Johal SS, Lambert CP, Hammond J, James PD, Borriello SP, Mahida YR. Colonic IgA producing cells and macrophages are reduced in recurrent and non-recurrent Clostridium difficile associated diarrhoea. JClin Pathol. 2004;57(9):973-979.

57. Atarashi K, et al. Treg induction by a rationally selected mixture of Clostridia strains from the human microbiota. Nature. 2013;500 (7461):232-236.

58. Gaboriau-Routhiau V, et al. The key role of segmented filamentous bacteria in the coordinated maturation of gut helper $\mathrm{T}$ cell responses. Immunity. 2009;31(4):677-689.

59. Russell SL, et al. Early life antibiotic-driven changes in microbiota enhance susceptibility to allergic asthma. EMBO Rep. 2012;13(5):440-447.

60. Human Microbiome Project Consortium. Structure, function and diversity of the healthy human microbiome. Nature. 2012;486(7402):207-214.

61. Hopkins MJ, Macfarlane GT. Changes in predominant bacterial populations in human faeces with age and with Clostridium difficile infection. JMed Microbiol. 2002;51(5):448-454.

62. Manges AR, et al. Comparative metagenomic study of alterations to the intestinal microbiota and risk of nosocomial Clostridum difficile-associated disease. J Infect Dis. 2010;202(12):1877-1884 .

63. Rea MC, et al. Clostridium difficile carriage in elderly subjects and associated changes in the intestinal microbiota. JClin Microbiol. 2012;50(3):867-875.

64. Antharam VC, et al. Intestinal dysbiosis and depletion of butyrogenic bacteria in Clostridium difficile infection and nosocomial diarrhea. JClin Microbiol. 2013;51(9):2884-2892.

65. Schubert AM, et al. Microbiome data distinguish patients with Clostridium difficile infection and non-C. difficile-associated diarrhea from healthy controls. MBio. 2014;5(3):e01021-14.

66. Lawley TD, et al. Antibiotic treatment of clostridium difficile carrier mice triggers a supershedder 
state, spore-mediated transmission, and severe disease in immunocompromised hosts. Infect Immun. 2009;77(9):3661-3669.

67. Buffie CG, et al. Profound alterations of intestinal microbiota following a single dose of clindamycin results in sustained susceptibility to Clostridium difficile-induced colitis. Infect Immun. 2012;80(1):62-73.

68. Reeves AE, Theriot CM, Bergin IL, Huffnagle GB, Schloss PD, Young VB. The interplay between microbiome dynamics and pathogen dynamics in a murine model of Clostridium difficile infection. Gut Microbes. 2011;2(3):145-158.

69. Reeves AE, Koenigsknecht MJ, Bergin IL, Young VB. Suppression of Clostridium difficile in the gastrointestinal tracts of germfree mice inoculated with a murine isolate from the family Lachnospiraceae. Infect Immun. 2012;80 (11):3786-3794.

70. Pépin J, Routhier S, Gagnon S, Brazeau I. Management and outcomes of a first recurrence of Clostridium difficile-associated disease in Quebec, Canada. Clin Infect Dis. 2006;42(6):758-764.

71. Drekonja DM, et al. Antimicrobial use and risk for recurrent Clostridium difficile infection. $\mathrm{Am} J$ Med.2011;124(11):e1081-e1087.

72. Chang JY, et al. Decreased diversity of the fecal Microbiome in recurrent Clostridium difficile-associated diarrhea. J Infect Dis. 2008;197(3):435-438.

73. Gough E, Shaikh H, Manges AR. Systematic review of intestinal microbiota transplantation (fecal bacteriotherapy) for recurrent Clostridium difficile infection. Clin Infect Dis. 2011;53(10):994-1002.

74. Tvede M, Rask-Madsen J. Bacteriotherapy for chronic relapsing Clostridium difficile diarrhoea in six patients. Lancet. 1989;1(8648):1156-1160.

75. Khoruts A, Dicksved J, Jansson JK, Sadowsky MJ. Changes in the composition of the human fecal microbiome after bacteriotherapy for recurrent Clostridium difficile-associated diarrhea. J Clin Gastroenterol. 2010;44(5):354-360.

76. van Nood E, et al. Duodenal infusion of donor feces for recurrent Clostridium difficile. $N$ EnglJ Med. 2013;368(5):407-415.

77. Hamilton MJ, Weingarden AR, Unno T, Khoruts A, Sadowsky MJ. High-throughput DNA sequence analysis reveals stable engraftment of gut microbiota following transplantation of previously frozen fecal bacteria. Gut Microbes. 2013;4(2):125-135.

78. Song Y, et al. Microbiota dynamics in patients treated with fecal microbiota transplantation for recurrent Clostridium difficile infection. PLoS One. 2013;8(11):e81330.

79. Angelberger S, et al. Temporal bacterial community dynamics vary among ulcerative colitis patients after fecal microbiota transplantation. Am J Gastroenterol. 2013;108(10):1620-1630.

80. Fuentes S, et al. Reset of a critically disturbed microbial ecosystem: faecal transplant in recurrent Clostridium difficile infection [published online ahead of print February 27, 2014]. ISME J. doi:10.1038/ismej.2014.13.

81. Young VB, Schmidt TM. Antibiotic-associated diarrhea accompanied by large-scale alterations in the composition of the fecal microbiota. JClin Microbiol. 2004;42(3):1203-1206.

82. Seekatz AM, et al. Recovery of the gut microbiome following fecal microbiota transplantation. MBio. 2014;5(3):e00893-e00814.

83. Johnson S, Schriever C, Galang M, Kelly CP, Gerding DN. Interruption of recurrent Clostridium difficile-associated diarrhea episodes by serial therapy with vancomycin and rifaximin. Clin Infect Dis. 2007;44(6):846-848.

84. Johnson S, Gerding DN. Fidaxomicin "chaser" regimen following vancomycin for patients with multiple Clostridium difficile recurrences. Clin Infect Dis. 2013;56(2):309-310.

85. Louie TJ, Emery J, Krulicki W, Byrne B, Mah M. OPT-80 eliminates Clostridium difficile is sparing of bacteroides species during treatment of C. difficile infection. Antimicrob Agents Chemother. 2009;53(1):261-263.

86. Jump RL, Li Y, Pultz MJ, Kypriotakis G, Donskey CJ. Tigecycline exhibits inhibitory activity against Clostridium difficile in the colon of mice and does not promote growth or toxin production. Antimicrob Agents Chemother. 2011;55(2):546-549.

87. Larson KC, Belliveau PP, Spooner LM. Tigecycline for the treatment of severe Clostridium difficile infection. Ann Pharmacother. 2011; 45(7-8):1005-1010.

88. Bassis CM, Theriot CM, Young VB. Alteration of the murine gastrointestinal microbiota by tigecycline leads to increased susceptibility to Clostridium difficile infection. Antimicrob Agents Chemother. 2014;58(5):2767-2774.

89. Kyne L, Warny M, Qamar A, Kelly CP. Asymptomatic carriage of Clostridium difficile and serum levels of IgG antibody against toxin A. N Engl J Med. 2000;342(6):390-397.

90. Kyne L, Warny M, Qamar A, Kelly CP. Association between antibody response to toxin $\mathrm{A}$ and protection against recurrent Clostridium difficile diarrhoea. Lancet. 2001;357(9251):189-193.

91. Gerding DN, Johnson S. Management of Clostridium difficile infection: thinking inside and outside the box. Clin Infect Dis. 2010;51(11):1306-1313.

92. Baines SD, Freeman J, Wilcox MH. Tolevamer is not efficacious in the neutralization of cytotoxin in a human gut model of Clostridium difficile infection. Antimicrob Agents Chemother. 2009;53(5):2202-2204.

93. Lawley TD, et al. Targeted restoration of the intestinal microbiota with a simple, defined bacteriotherapy resolves relapsing Clostridium difficile disease in mice. PLoS Pathog. 2012;8(10):e1002995.

94. Petrof EO, et al. Stool substitute transplant therapy for the eradication of Clostridium difficile infection: 'RePOOPulating' the gut. Microbiome. 2013;1(1):3.

95. Shim JK, Johnson S, Samore MH, Bliss DZ, Gerding DN. Primary symptomless colonisation by Clostridium difficile and decreased risk of subsequent diarrhoea. Lancet. 1998;351(9103):633-636.

96. Nagaro KJ, et al. Nontoxigenic Clostridium difficile protects hamsters against challenge with historic and epidemic strains of toxigenic $\mathrm{BI} /$ NAP1/027 C. difficile. Antimicrob Agents Chemother. 2013;57(11):5266-5270.

97. Villano SA, Seiberling M, Tatarowicz W, Monnot-Chase E, Gerding DN. Evaluation of an oral suspension of VP20621, spores of nontoxigenic Clostridium difficile strain M3, in healthy subjects. Antimicrob Agents Chemother. 2012;56(10):5224-5229.

98. Skraban J, Dzeroski S, Zenko B, Mongus D, Gangl S, Rupnik M. Gut microbiota patterns associated with colonization of different Clostridium difficile ribotypes. PLoS One. 2013;8(2):e58005. 